\title{
Consensus of Heterogeneous Multiagent Systems with Arbitrarily Bounded Communication Delay
}

\author{
Xue Li, Huai Wu, and Yikang Yang \\ School of Aeronautics and Astronautics, University of Electronic Science and Technology of China, Chengdu 611731, China \\ Correspondence should be addressed to Xue Li; lixue.1981@uestc.edu.cn
}

Received 11 July 2016; Revised 16 January 2017; Accepted 6 February 2017; Published 11 April 2017

Academic Editor: Damijan Markovic

Copyright (C) 2017 Xue Li et al. This is an open access article distributed under the Creative Commons Attribution License, which permits unrestricted use, distribution, and reproduction in any medium, provided the original work is properly cited.

\begin{abstract}
This paper focuses on the consensus problem of high-order heterogeneous multiagent systems with arbitrarily bounded communication delays. Through the method of nonnegative matrices, we get a sufficient consensus condition for the systems with dynamically changing topology. The results of this paper show, even when there are arbitrarily bounded communication delays in the systems, all agents can reach a consensus no matter whether there are spanning trees for the corresponding communication graphs at any time.
\end{abstract}

\section{Introduction}

In the past few years, consensus problems for multiagent systems have had a significant impact on many fields, including wireless sensor networks, mobile robot formation mission, and formation flying for satellite. Consensus means the outputs of the agents that are spatially distributed can reach a common value. The consensus problems have attracted much attention from academia, and there have been a great number of results investigating the consensus problems for networks of dynamic agents [1-8].

In reality, for the influences of the finite speeds of transmission and spreading, multiagent systems are often restricted to communication delays. There have been many works dealing with the study of consensus problems with time-delays [9-17]. For example, [9] investigated the consensus problem with time-delay, obtained the sufficient and necessary condition, and gave the largest tolerable input delay to guarantee the consensus. In [10], Lin and Jia extended the results in [9] to second-order system through the method of linear matrix inequalities. In [11], an upper bound for delay tolerance is obtained for the high-order system when all eigenvalues of each agent are in the closed left half plane. In [12], Zhou and Lin used output feedback protocols to investigate the consensus problem when the time-delays are constant and exactly known even if arbitrarily bounded. The approaches to analyse the consensus problems for multiagent systems fall into three major groups: the Lyapunov functions, the frequency-domain analysis, and the method based on the properties of nonnegative matrices [3, 6, 18-25]. There have been a series of papers highlighting first-order, secondorder, high-order, and mixed-order multiagent systems, and great progress has been made in this field. In [3], C.-L. Liu and F. Liu researched the consensus problem of discretetime heterogeneous multiagent systems composed of firstorder agents and second-order agents through the method of nonnegative matrices. In [24], Zheng and Wang proposed two classes of consensus protocols for heterogeneous multiagent systems with and without velocity measurements. However, no clear advancement has so far been seen in the field of highorder heterogeneous multiagent systems.

In this paper, we investigate the consensus problem of high-order heterogeneous multiagent systems with arbitrarily bounded time-delays through the method of nonnegative matrices. Under some assumptions, we get a sufficient consensus condition for the multiagent systems with dynamically changing topology and arbitrarily bounded delays.

Through this article, we let $\mathbb{R}^{m}$ and $\mathbb{Z}_{+}$represent $m$ dimensional real vector space and the set of nonnegative integers, respectively. $I_{n} \in \mathbb{R}^{n \times n}$ is the identity matrix. $\mathbf{1}_{n}=$ $[1,1, \ldots, 1]^{T} \in \mathbb{R}^{n} . \otimes$ is the Kronecker product. 


\section{Graph Theory}

A directed graph $\mathscr{G}$ is composed of a vertex set $\mathscr{V}=$ $\left\{s_{1}, \ldots, s_{n}\right\}$, an arc set $\varepsilon \subseteq \mathscr{V} \times \mathscr{V}$, and a weighted adjacency matrix $\mathscr{A}=\left[a_{i j}\right]$, denoted by $\mathscr{G}=(\mathscr{V}, \mathcal{\varepsilon}, \mathscr{A})$. The node indexes belong to a finite index set $\mathscr{L}=\{1,2, \ldots, n\}$. If there is a directed edge from node $s_{i}$ to node $s_{j}$, then $s_{i}$ and $s_{j}$ are called the tail and the head of $\left(s_{i}, s_{j}\right)$, respectively. The neighbors of agent $i$ are denoted by $N_{i}=\left\{s_{j} \in \mathscr{V}:\left(s_{i}, s_{j}\right) \in\right.$ $\varepsilon\}$. The adjacency elements associated with the edges of the digraph are defined as $a_{i i}=0$ and $a_{i j}>0$ if $e_{i j} \in \varepsilon$. The Laplacian matrix of $\mathscr{G}(\mathscr{V}, \varepsilon, \mathscr{A})$ is defined as $L=\Delta-\mathscr{A}$, where $\Delta=\left[\Delta_{i j}\right]$ is a diagonal matrix with $\Delta_{i i}=\sum_{j=1}^{n} a_{i j}$. By the definition of $L$ we can get $L \mathbf{1}_{n}=\mathbf{0}$. A directed path is a sequence of ordered edges of the form $\left(s_{i_{1}}, s_{i_{2}}\right),\left(s_{i_{2}}, s_{i_{3}}\right), \ldots$, where $s_{i_{j}} \in v$ in a directed graph. A directed graph is said to be strongly connected if and only if there is a directed path from every node to every other node. A directed graph has a spanning tree if there is a node such that there exists a directed path from every other node to this node.

Given $Q=\left[q_{i j}\right] \in \mathbb{R}^{n \times r}$, when all of its elements $q_{i j}$ are nonnegative there we say $Q$ is nonnegative and $Q \geq 0$. If $Q \in \mathbb{R}^{n \times n}$ is nonnegative and it satisfies $Q \mathbf{1}=\mathbf{1}$, then $Q$ is stochastic. When a stochastic matrix $Q$ satisfies $\lim _{k \rightarrow+\infty} Q^{k}=$ $\mathbf{1} f^{T}$, where $f \in \mathbb{R}^{n}$, then $Q$ is stochastic, indecomposable, and aperiodic (SIA).

\section{Model}

Suppose that discrete-time heterogeneous multiagent system consists of first-order agents, second-order agents, thirdorder agents, until $l$ th-order agents and the total number of agents is $n$, where $n=n_{1}+n_{2}+\cdots+n_{l}$, and $n_{i}(i=1,2, \ldots, l-1)$ denotes the quantity of the $i$ th-order agents.

Suppose the dynamics of the first-order agents are

$$
\xi_{i 1}^{(0)}(k+1)=\xi_{i 1}^{(0)}(k)+u_{i_{1}}(k) T, \quad i_{1}=1,2, \ldots, n_{1},
$$

where $\xi_{i 1}^{(0)} \in \mathbb{R}$ is the position, $u_{i_{1}} \in \mathbb{R}$ is the control input, and $T>0$ is the sample time. To solve the agreement problems, the protocol with communication delays is proposed for the first-order agents as follows:

$$
u_{i_{1}}(k)=-\sum_{j \in N_{i_{1}}} a_{i_{1} j}(k)\left(\xi_{i 1}^{(0)}(k)-\xi_{j}^{(0)}\left(k-\tau_{i_{1} j}(k)\right)\right) .
$$

The communication delay $0 \leq \tau_{i_{1} j}(k) \leq \tau_{\max }$ corresponds to the information flow from agent $j$ to agent $i_{1}$ and $a_{i_{1} j}>0, j \in$ $N_{i_{1}}(k)$, is the coupling weight chosen from any finite set and $N_{i_{1}}(k)$ is the neighboring agents of agent $i_{1}$.

Suppose the dynamics of the second-order agents are

$$
\begin{aligned}
& \xi_{i_{2}}^{(0)}(k+1)=\xi_{i_{2}}^{(0)}(k)+\xi_{i_{2}}^{(1)}(k) T, \\
& \xi_{i_{2}}^{(1)}(k+1)=\xi_{i_{2}}^{(1)}(k)+u_{i_{2}}(k) T,
\end{aligned}
$$

where $i_{2}=n_{1}+1, n_{1}+2, \ldots, n_{1}+n_{2} \cdot \xi_{i_{2}}^{(0)} \in \mathbb{R}$ is the position, $\xi_{i_{2}}^{(1)} \in \mathbb{R}$ is the velocity, and $u_{i_{2}} \in \mathbb{R}$ is the control input. To solve the agreement problems, the protocol with communication delays is proposed for the second-order agents as follows:

$$
\begin{aligned}
u_{i_{2}}(k) & \\
= & -p_{1} \xi_{i_{2}}^{(1)}(k) \\
& -\sum_{j \in N_{i_{2}}(k)} a_{i_{2} j}(k)\left(\xi_{i_{2}}^{(0)}(k)-\xi_{j}^{(0)}(k)\left(k-\tau_{i_{2} j}(k)\right)\right) .
\end{aligned}
$$

The communication delay $0 \leq \tau_{i_{2} j}(k) \leq \tau_{\max }$ corresponds to the information flow from agent $j$ to agent $i_{2}$ and $a_{i_{2} j}>0, j \in$ $N_{i_{2}}(k)$, is the coupling weight chosen from any finite set and $N_{i_{2}}(k)$ is the neighboring agents of agent $i_{2} \cdot p_{1}>0$ is control parameter.

Similarly, we suppose the dynamics of $m$ th-order agents are

$$
\begin{aligned}
& \xi_{i_{m}}^{(0)}(k+1)=\xi_{i_{m}}^{(0)}(k)+\xi_{i_{m}}^{(1)}(k) T \\
& \xi_{i_{m}}^{(m-2)}(k+1)=\xi_{i_{m}}^{(m-2)}(k)+\xi_{i_{m}}^{(m-1)}(k) T \\
& \xi_{i_{m}}^{(m-1)}(k+1)=\xi_{i_{m}}^{(m-1)}(k)+u_{i_{m}}(k) T,
\end{aligned}
$$

where $m=3,4, \ldots, l, i_{m}=\sum_{z=1}^{m-1} n_{z}+1, \sum_{z=1}^{m-1} n_{z}+$ $2, \ldots, \sum_{z=1}^{m} n_{z} . \xi_{i m}^{(j)} \in \mathbb{R}$ is the $j$ th variable of $\xi_{i m}, j=$ $0,1, \ldots, m-1$, and $u_{i_{m}}(k) \in \mathbb{R}$ is the control input. To solve the agreement problems, the protocol with communication delays is proposed for $m$ th agents as follows:

$$
\begin{aligned}
u_{i_{m}}(k)= & -\sum_{j=1}^{m-1} p_{j} \xi_{i_{m}}^{(j)}(k) \\
& -\sum_{s_{j} \in N_{i_{m}}(k)} a_{i_{m} j}(k)\left(\xi_{i_{m}}^{(0)}(k)-\xi_{j}^{(0)}\left(k-\tau_{i_{m} j}\right)\right) .
\end{aligned}
$$

The communication delay $0 \leq \tau_{i_{m} j}(k) \leq \tau_{\max }$ corresponds to the information flow from agent $j$ to agent $i_{m}$ and $a_{i_{m} j}>$ $0, j \in N_{i_{m}}(k)$, is the coupling weight chosen from any finite set and $N_{i_{m}}(k)$ is the neighboring agents of agent $i_{m} \cdot p_{j}$ is control parameter and $p_{j}>0$ for all $j=1,2, \ldots, m-1$. It is obvious to see that when the states of agents satisfy the following, the high-order heterogeneous multiagent systems in this paper can reach consensus:

$$
\lim _{k \rightarrow+\infty}\left[\xi_{i}^{(a)}(k)-\xi_{j}^{(a)}(k)\right]=0
$$

where $i, j \in \mathscr{L}$, agent $i$ belongs to the $m$ th-order agents, and agent $j$ belongs to the $n$ th-order agents. $a$ is any nonnegative integer which satisfies $a<m, a<n$.

Let $\xi(k)=\left[\xi_{1}^{T}(k), \xi_{2}^{T}(k), \ldots, \xi_{l}^{T}(k)\right]^{T}$, where $\xi_{1}=\left[\xi_{1}^{(0)}\right.$, $\left.\xi_{2}^{(0)}, \ldots, \xi_{n_{1}}^{(0)}\right], \xi_{2}=\left[\xi_{n_{1}+1}^{(0)}, \xi_{n_{1}+1}^{(1)}, \xi_{n_{1}+2}^{(0)}, \xi_{n_{1}+2}^{(1)}, \ldots, \xi_{n_{1}+n_{2}}^{(0)}, \xi_{n_{1}+n_{2}}^{(1)}\right]$, 
$\ldots, \xi_{l}=\left[\xi_{c+1}^{(0)}, \xi_{c+1}^{(1)}, \ldots, \xi_{c+1}^{(l-1)}, \ldots, \xi_{c+n_{l}}^{(0)}, \xi_{c+n_{l}}^{(1)}, \ldots, \xi_{c+n_{l}}^{(l-1)}\right], c=$ $\sum_{i=1}^{l-1} n_{i}$, and

$\bar{A}_{k}$

$$
=\left(\begin{array}{ccccccc}
1 & T & \mathbf{0} & \mathbf{0} & \cdots & \mathbf{0} & \mathbf{0} \\
\mathbf{0} & 1 & T & \mathbf{0} & \cdots & \mathbf{0} & \mathbf{0} \\
\mathbf{0} & \mathbf{0} & 1 & T & \cdots & \mathbf{0} & \mathbf{0} \\
\vdots & \vdots & \vdots & \vdots & \ddots & \vdots & \vdots \\
\mathbf{0} & \mathbf{0} & \mathbf{0} & \mathbf{0} & \cdots & 1 & T \\
\mathbf{0} & -p_{1} T & -p_{2} T & -p_{3} T & \cdots & -p_{k-2} T & 1-p_{k-1} T
\end{array}\right)
$$

$$
\in \mathbb{R}^{k \times k} \text {. }
$$

Here we set $A_{1}=I_{n_{1}}$ and $A_{k}=\operatorname{diag}\left\{\bar{A}_{k}, \bar{A}_{k}, \ldots, \bar{A}_{k}\right\} \in$ $\mathbb{R}^{k n_{k} \times k n_{k}}$, where $k=2,3, \ldots, l$. So we define $A=\operatorname{diag}\left\{A_{1}\right.$, $\left.A_{2}, \ldots, A_{l}\right\}$ :

$$
L(k)=\left(\begin{array}{cccc}
\bar{L}_{1}(k) & -L_{12}(k) & \cdots & -L_{1 l}(k) \\
-L_{21}(k) & \bar{L}_{2}(k) & \cdots & -L_{2 l}(k) \\
\vdots & \vdots & \ddots & \vdots \\
-L_{l 1}(k) & -L_{l 2}(k) & \cdots & -\bar{L}_{l}(k)
\end{array}\right),
$$

where $L(k)$ denotes the Laplacian matrix of the graph $\mathscr{G}$, $\bar{L}_{i}(k)=L_{i}(k)+D_{i}(k), L_{i}(k)$ is the Laplacian matrix of $i$ thorder agents, and $D_{i}(k)=\operatorname{diag}\left\{\sum_{j \in N_{i}^{o}} a_{i j}(k), i=1,2, \ldots, c\right\}$, $c=\sum_{i=1}^{l} n_{i} . N_{i}^{o}$ denotes agent $i$ 's neighboring agents except all the agents which are the same order as agent $i . L_{i j}(k)$ are the adjacency relations of $i$ th-order agents to $j$ th-order agents:

$$
B_{i j}=\left(\begin{array}{cccc}
0 & 0 & \cdots & 0 \\
0 & 0 & \cdots & 0 \\
\vdots & \vdots & \ddots & \vdots \\
T & 0 & \cdots & 0
\end{array}\right) \in \mathbb{R}^{i \times j}, \quad i, j=1,2, \ldots, l,
$$

$$
=\left(\begin{array}{cccc}
\bar{L}_{1}(k) \otimes B_{11} & -L_{12}(k) \otimes B_{12} & \cdots & -L_{1 l}(k) \otimes B_{1 l} \\
-L_{21}(k) \otimes B_{21} & \bar{L}_{2}(k) \otimes B_{22} & \cdots & -L_{2 l}(k) \otimes B_{2 l} \\
\vdots & \vdots & \ddots & \vdots \\
-L_{1 l}(k) \otimes B_{l 1} & -L_{l 2}(k) \otimes B_{l 2} & \cdots & \bar{L}_{l}(k) \otimes B_{l l}
\end{array}\right) .
$$

The network of the multiagent system is

$$
\begin{aligned}
\xi(k+1)= & {\left[A-\left(L_{0}(k)-C_{0}(k)\right)\right] \xi(k) } \\
& +C_{1}(k) \xi(k-1)+\cdots \\
& +C_{\tau_{\max }}(k) \xi\left(k-\tau_{\max }\right),
\end{aligned}
$$

where $L_{0}(k)=\operatorname{diag}(\bar{L}(k))$ and the $i j$ th element of $C_{m}(k)(m=$ $\left.0,1, \ldots, \tau_{\max }\right)$ is equal to zero or the weight of the edge $e_{i j}$ if $\tau_{i j}=m$. And we can see that $\bar{L}(k)=L_{0}(k)-\sum_{m=0}^{\tau_{\max }} C_{m}(k)$.

\section{Main Results}

Assumption 1. Consider

$$
\begin{aligned}
H_{m} & >T, \\
k_{i 1} & >d_{\max } T \prod_{n=1}^{i-1} H_{n}, \\
k_{i j} & >0 \\
1+k_{i i} & >0
\end{aligned}
$$

for $m=1,2, \ldots, l, i=2,3, \ldots, l$, and $j=1,2, \ldots, i-1$, where $d_{\text {max }}$ denotes the largest entry of all possible $L(k)$.

Lemma 2 (see [26]). Let $P_{1}, P_{2}, \ldots, P_{k} \in R^{q \times q}$ be a finite set of SIA matrices with the property that, for each sequence $P_{i_{1}}$, $P_{i_{2}}, \ldots, P_{i_{j}}$ of positive length, the matrix product $P_{i_{j}} P_{i_{j-1}} \ldots P_{i_{1}}$ is SIA. Then, for each infinite sequence $P_{i_{1}}, P_{i_{2}}$, there exists a vector $f \in \mathbb{R}^{q}$ such that $\prod_{j=1}^{\infty} P_{i_{j}}=\mathbf{1} f^{T}$.

Theorem 3. Suppose that there exists an infinite strictly increasing $k_{\alpha}$ with $k_{0}=0$ and $0<k_{\alpha+1}-k_{\alpha} \leq \beta, \alpha, \beta \in \mathbb{Z}_{+}$. If the union of $\left\{\mathscr{G}\left(k_{\alpha}\right), \mathscr{G}\left(k_{\alpha}+1\right), \ldots, \mathscr{G}\left(k_{\alpha+1}-1\right)\right\}$ has a spanning tree, then the agents in systems (1), (3), and (5) converge to a stationary consensus asymptotically.

Proof. Let $\phi_{i}(k)=\Gamma_{i} \xi_{i}(k), i=1,2, \ldots, l$, and $\phi(k)=\left[\phi_{1}^{T}(k)\right.$, $\left.\phi_{2}^{T}(k), \ldots, \phi_{n}^{T}(k)\right]$ :

$$
\Gamma_{i i}=\left(\begin{array}{ccccc}
1 & 0 & 0 & \cdots & 0 \\
1 & H_{1} & 0 & \cdots & 0 \\
1 & \sum_{j_{0}=1}^{2} H_{j_{0}} & H_{1} H_{2} & \cdots & 0 \\
\vdots & \vdots & \vdots & \ddots & 0 \\
1 & \sum_{j_{0}=1}^{i-1} H_{j_{0}} & \sum_{j_{0}=1}^{l-2} \prod_{j_{1}=j_{0}+1}^{i-1} H_{j_{0} j_{1}} & \cdots & \prod_{j_{0}=1}^{i-1} H_{j_{0}}
\end{array}\right)
$$

$\Gamma_{1}=I_{n_{1}}$, and $\Gamma_{i}=\operatorname{diag}\left\{\Gamma_{i i}, \Gamma_{i i}, \ldots, \Gamma_{i i}\right\} \in \mathbb{R}^{\left(n_{i} \times i\right) \times\left(n_{i} \times i\right)}, i=$ $2,3, \ldots, l$ for some positive constants $H_{i} \in \mathbb{R}$.

Let

$M(k)$

$$
=\left(\begin{array}{cccc}
\bar{L}_{1}(k) \otimes F_{11} & -L_{12}(k) \otimes F_{12} & \cdots & -L_{1 l}(k) \otimes F_{1 l} \\
-L_{21}(k) \otimes F_{21} & \bar{L}_{2}(k) \otimes F_{22} & \cdots & -A_{2 l}(k) \otimes F_{2 l} \\
\vdots & \vdots & \ddots & \vdots \\
-L_{l 1}(k) \otimes F_{l 1} & -L_{l 2}(k) \otimes F_{l 2} & \cdots & \bar{L}_{l}(k) \otimes F_{l l}
\end{array}\right) .
$$


Then we can transform system (11) into the following system:

$$
\begin{aligned}
\phi(k+1)= & {\left[E-\left(M_{0}(k)-\bar{C}_{0}(k)\right)\right] \phi(k) } \\
& +\bar{C}_{1}(k) \phi(k-1)+\cdots \\
& +\bar{C}_{\tau_{\max }}(k) \phi\left(k-\tau_{\max }\right),
\end{aligned}
$$

where $F_{i j}=B_{i j} \prod_{k=1}^{i-1} H_{k}, F_{1 j}=B_{1 j}, i=2,3, \ldots, l$ and $j=$ $1,2, \ldots, l, M_{0}(k)=\operatorname{diag}(M(k))$, and the $i j$ th element of $\bar{C}_{m}(k)\left(m=0,1,2, \ldots, \tau_{\max }\right)$ is either zero or equal to the weight of the element of $m_{i j}$ if $\tau_{i j}=m$ :

$$
\begin{aligned}
E_{i i} & =\left[\begin{array}{cccccc}
1-\frac{T}{H_{1}} & \frac{T}{H_{1}} & 0 & \cdots & 0 & 0 \\
0 & 1-\frac{T}{H_{2}} & \frac{T}{H_{2}} & \cdots & 0 & 0 \\
\vdots & \vdots & \vdots & \ddots & \vdots & \vdots \\
0 & 0 & 0 & \cdots & 1-\frac{T}{H_{i-1}} & \frac{T}{H_{i-1}} \\
k_{i 1} & k_{i 2} & k_{i 3} & \cdots & k_{i, i-1} & k_{i i}+1
\end{array}\right] \\
& \in \mathbb{R}^{i \times i}, \quad i=2,3, \ldots, l .
\end{aligned}
$$

$E_{1}=I_{n_{1}}$ and $E_{i}=\operatorname{diag}\left\{E_{i i}, E_{i i}, \ldots, E_{i i}\right\} \in \mathbb{R}^{\left(n_{i} \times i\right) \times\left(n_{i} \times i\right)}, i=$ $2,3, \ldots, l$ and $E=\operatorname{diag}\left\{E_{1}, E_{2}, \ldots, E_{l}\right\}$ for constants $k_{i j} \in \mathbb{R}$.

Define $Y(k)=\left[\phi^{T}(k), \phi^{T}(k-1), \ldots, \phi^{T}\left(k-\tau_{\max }\right)\right]^{T}$. Then system (15) can be transformed into

$$
Y(k+1)=\Phi(k) Y(k)
$$

where $\Phi(k)$ is defined as follows:

$$
\begin{aligned}
& \Phi(k) \\
& =\left(\begin{array}{ccccc}
E-M_{0}(k)+\bar{C}_{0}(k) & \bar{C}_{1}(k) & \cdots & \bar{C}_{\tau_{\max -1}}(k) & \bar{C}_{\tau_{\max }}(k) \\
I & \mathbf{0} & \cdots & \mathbf{0} & \mathbf{0} \\
\mathbf{0} & I & \cdots & \mathbf{0} & \mathbf{0} \\
\mathbf{0} & \vdots & \ddots & \vdots & \vdots \\
\mathbf{0} & \mathbf{0} & \cdots & I & \mathbf{0}
\end{array}\right) .
\end{aligned}
$$

We can see $E_{i i}=\Gamma_{i i} \bar{A}_{i} \Gamma_{i i}^{-1}, i=3,4, \ldots, l$, and thus

$$
\begin{aligned}
& {\left[k_{i 1}, k_{i 2}, \ldots, k_{i i}+1\right]} \\
& \quad=\left[1 \sum_{j_{0}=1}^{i-1} H_{j_{0}} \sum_{j_{0}=1}^{i-2} \prod_{j_{1}=j_{0}+1}^{i-1} H_{j_{0} j_{1}} \cdots \prod_{j_{0}=1}^{i-1} H_{j_{0}}\right]\left[\left(\bar{A}_{i}\right.\right.
\end{aligned}
$$

$$
\begin{aligned}
& -I)+I] \Gamma_{i i}^{-1} \\
& =\left[1 \sum_{j_{0}=1}^{i-1} H_{j_{0}} \sum_{j_{0}=1}^{i-2} \prod_{j_{1}=j_{0}+1}^{i-1} H_{j_{0} j_{1}} \cdots \prod_{j_{0}=1}^{i-1} H_{j_{0}}\right]\left(\bar{A}_{i}\right. \\
& -I) \Gamma_{i i}^{-1}+\left[0_{i-1}^{T}, 1\right]=\left[0,\left(1-p_{1} \prod_{m=1}^{i-1} H_{m}\right) T,\right. \\
& \left(\sum_{m=1}^{i-1} H_{m}-p_{2} \prod_{m=1}^{i-1} H_{m}\right) T, \sum_{j_{0}=1}^{i-2} \prod_{j_{1}=j_{0}+1}^{i-1} H_{j_{0}} H_{j_{1}} \\
& -p_{3} \prod_{m=1}^{i-1} H_{m} T, \ldots,\left(\sum_{j_{0}=1}^{i-1} \prod_{m=1, m \neq j_{0}}^{i-1} H_{m}\right) \\
& \left.-p_{i-1} \prod_{m=1}^{i-1} H_{m} T\right] \Gamma_{i i}^{-1}+\left[0_{i-1}^{T}, 1\right] .
\end{aligned}
$$

Due to the fact that the elements of the first column of $\Gamma_{i i}$ are all 1 , and $\Gamma_{i i} \Gamma_{i i}^{-1}=I_{i}$, so we can easily get that only the first row sum of $\Gamma_{i i}^{-1}$ is 1 and the other row sums of $\Gamma_{i i}^{-1}$ are all 0 . Thus we can see $\left[k_{i 1}, k_{i 2}, \ldots, k_{i i}\right] \mathbf{1}=0$. Considering that all row sums of $L(k)$ are 0 , so $[E-M(k)] \mathbf{1}=\mathbf{1}$. Considering that $M_{0}(k)=M(k)-\sum_{m=0}^{\tau_{\max }} \bar{C}_{m}(k)$, so $\Phi(k) \mathbf{1}=\mathbf{1}$. Under Assumption 1, there is no negative element in $\Phi(k)$. So from the above we can get $\Phi(k)$ is a stochastic matrix.

There we assume that $\alpha_{k}$ is the largest positive integer which satisfies $k_{\alpha_{k}} \leq k$ for each $k \geq 0$. So $Y(k+$ 1) $=\Phi(k) \cdots \Phi\left(k_{\alpha_{k}}\right) \prod_{\alpha=0}^{\alpha_{k}-1} \Delta(\alpha) Y(0)$, and $\Delta(\alpha)=\Phi\left(k_{\alpha+1}-\right.$ 1) $\Phi\left(k_{\alpha+1}-2\right) \cdots \Phi\left(k_{\alpha}\right)$. Note that $k_{\alpha+1}-k_{\alpha} \leq \eta$ and the union of $\left\{\mathscr{G}\left(k_{\alpha}\right), \mathscr{G}\left(k_{\alpha}+1\right), \ldots, \mathscr{G}\left(k_{\alpha+1}-1\right)\right\}$ has a spanning tree. Under Assumption 1, similar to the proof of lemma 3 of [18], we can get $\Delta(\alpha)$ is SIA. And we can see the union of $\left\{\mathscr{G}\left(k_{\alpha}\right), \mathscr{G}\left(k_{\alpha}+1\right), \ldots, \mathscr{G}\left(k_{\alpha+j}-1\right)\right\}$ has spanning trees for some positive integer $j$. So it is easy to see that $\prod_{l=\alpha}^{\alpha+j-1} \Delta(l)$ is also SIA. For $0<k_{\alpha+1}-k_{\alpha} \leq \eta$ and $a_{i j}(k)$ are chosen from a finite set, the number of all possible $\Delta(j)$ is finite. Using Lemma 2, we can get that $\prod_{k=0}^{+\infty} \Delta(k)=\mathbf{1} f^{T}$ for some vector $f$. And each $\Phi(k)$ is a stochastic matrix. So

$$
\begin{aligned}
& \lim _{k \rightarrow+\infty} Y(k+1) \\
& =\lim _{k \rightarrow \infty} \Phi(k) \cdots \Phi\left(k_{m_{k}}\right) \prod_{m=0}^{m_{k}-1} \Delta(m) Y(0) \\
& =\lim _{k \rightarrow \infty} \Phi(k) \cdots \Phi\left(k_{m_{k}}\right) \mathbf{1} f^{T} Y(0)=\mathbf{1} f^{T} Y(0) .
\end{aligned}
$$

For $\lim _{k \rightarrow+\infty} Y(k+1)=\lim _{k \rightarrow+\infty} Y(k)$, we can get $\lim _{k \rightarrow+\infty} \phi_{i}(k)=\lim _{k \rightarrow+\infty} \Gamma_{i} \xi_{i}(k)=f^{T} Y(0)$ for all $i \in I$, which implies that $\lim _{k \rightarrow \infty} \xi_{i_{m}}^{(0)}(k)=f^{T} Y(0)$ and $\lim _{k \rightarrow \infty} \xi_{i_{m}}^{(n)}(k)=0$, for all $m=1,2, \ldots, l$ and $n \neq 0$, $n=1,2, \ldots, m-1$. This completes the proof. 


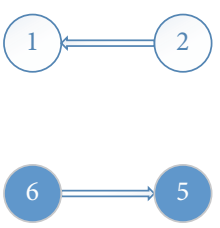

(a) Topology Ga
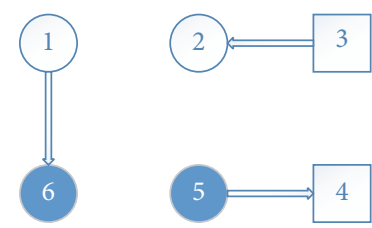

(b) Topology Gb

Figure 1: Two subfigures.

Remark 4. Similar to Proposition 1 of [18], when all parameters $H_{n}, n=1,2, \ldots, l-1$, are in the same value, it is to say that $H_{n}=H$, and we can always find parameters $p_{m}, m=1,2, \ldots, l-1$, satisfying Assumption 1 for any given parameters $H$ and $k_{i j}, i=2,3, \ldots, l, j=$ $1,2, \ldots, l-1$.

Remark 5. The existing results on heterogeneous multiagent systems, for example, $[3,24]$, only considered the systems composed of first-order and second-order agents, while the systems discussed in our paper do not only contain first-order and second-order agents but also contain high-order agents. The agents considered in the existing works contain at most only two variables (position states and velocity states), while the agents in this paper might contain no smaller than three variables (position states, velocity states, etc.). The variables of the agents are coupled in the form of integral. More kinds of agents with more variables might make the complexities of the whole multiagent systems increase in a geometrical rate. The approach of the existing works on the heterogeneous multiagent systems with first-order and second-order agents is based on the decoupling of the two variables and cannot be applied directly to the multiagent systems with high-order agents. Our approach is to introduce a model transformation to decouple different variables in each agent and decouple different kinds of agents so as to use the properties of the nonnegative matrices.

\section{Simulation Results}

In this section, by presenting some numerical simulations, we will verify the validity and correctness of the theoretical scheme. Considering the system is composed of six agents and the initial conditions of them are set randomly. The hollow circles represent the first-order agents, the squares represent the second-order agents, and the solid circles represent the third-order agents. We use the changing topology composed of $\mathrm{Ga}$ and $\mathrm{Gb}$, which starts at $\mathrm{Ga}$ and switches every $2 T$ s to the next state, which is shown in Figure 1. Obviously, the corresponding graphs $\mathrm{Ga}$ and $\mathrm{Gb}$ have no spanning tree, and the union of $\mathrm{Ga} \cup \mathrm{Gb}$ has a spanning tree. The sample time is $T=0.2 \mathrm{~s}$. Set $\tau_{12}=2, \tau_{23}=3, \tau_{34}=4$, $\tau_{45}=5, \tau_{56}=6, \tau_{61}=7, p_{3}=p_{4}=2, p_{51}=p_{61}=18$, and $p_{52}=p_{62}=6$, and then it can satisfy Assumption 1. The simulation results of each agent's third variables trajectories

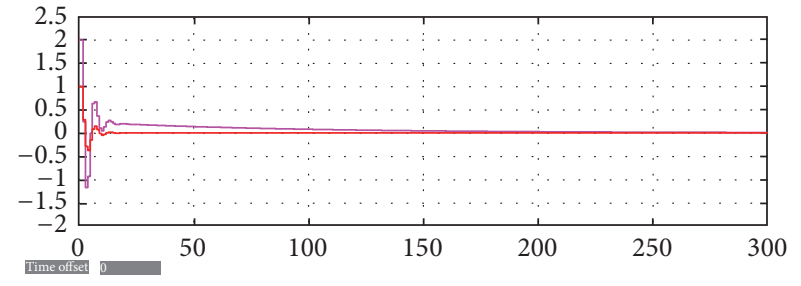

FIGURE 2: Acceleration trajectories of all agents.

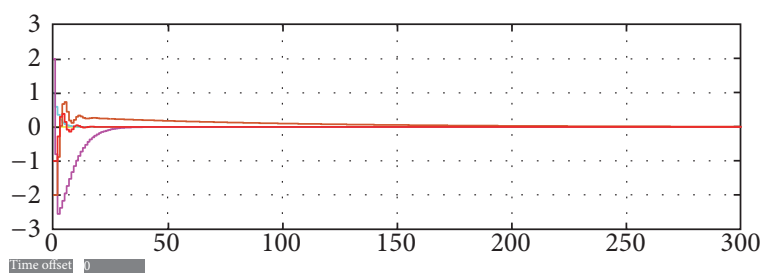

FIGURE 3: Velocity trajectories of all agents.

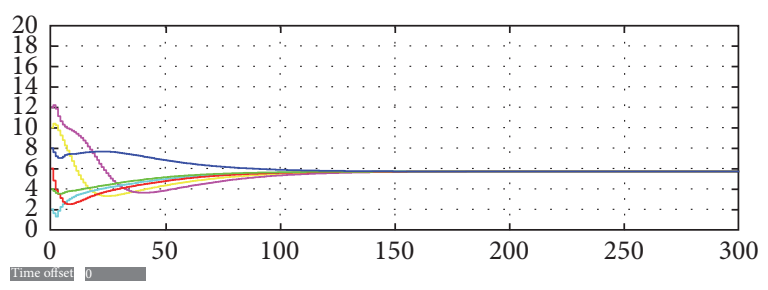

FIGURE 4: Position trajectories of all agents.

are shown in Figure 2, each agent's second variables trajectories are shown in Figure 3, and each agent's first variables trajectories are shown in Figure 4.

\section{Conclusion}

In this paper, we investigate the consensus problem for networks of high-order heterogeneous systems with timedelay. Through the method of the properties of the nonnegative matrices, we obtain a sufficient condition to guarantee the consensus of the directed heterogeneous network with arbitrarily bounded time-delay. Even for the high-order heterogeneous systems with dynamically changing topologies, it 
is shown that the output of the agents in the systems can reach consensus no matter whether there are spanning trees for the corresponding graphs.

\section{Conflicts of Interest}

The authors declare that they have no conflicts of interest.

\section{Acknowledgments}

This work was supported by the National Natural Science Foundation of China (61203080, 61573082, and 61304155), the National Program 863 of China (2015AA1528), and the National Program 973 of China (613237201406).

\section{References}

[1] "Consensus Algorithms for Double-integrator Dynamics," in Distributed Consensus in Multi-vehicle Cooperative Control, Communications and Control Engineering, pp. 77-104, Springer, London, England, 2008.

[2] F. Xiao and L. Wang, "State consensus for multi-agent systems with switching topologies and time-varying delays," International Journal of Control, vol. 79, no. 10, pp. 1277-1284, 2006.

[3] C.-L. Liu and F. Liu, "Stationary consensus of heterogeneous multi-agent systems with bounded communication delays," Automatica, vol. 47, no. 9, pp. 2130-2133, 2011.

[4] K. Srivastava and A. Nedić, "Distributed asynchronous constrained stochastic optimization," IEEE Journal on Selected Topics in Signal Processing, vol. 5, no. 4, pp. 772-790, 2011.

[5] P. Lin, W. Ren, and H. Gao, "Distributed velocity-constrained consensus of discrete-time multi-agent systems with nonconvex constraints, switching topologies, and delays," IEEE Transactions on Automatic Control, pp. 1-1, 2016.

[6] P. Lin, W. Ren, and J. A. Farrell, "Distributed continuoustime optimization: nonuniform gradient gains, finite-time convergence, and convex constraint set," IEEE Transactions on Automatic Control, 2016.

[7] P. Lin, W. Ren, and Y. Song, "Distributed multi-agent optimization subject to nonidentical constraints and communication delays," Automatica, vol. 65, pp. 120-131, 2016.

[8] P. Lin and Y. Jia, "Distributed rotating formation control of multi-agent systems," Systems \& Control Letters, vol. 59, no. 10, pp. 587-595, 2010.

[9] R. Olfati-Saber and R. M. Murray, "Consensus problems in networks of agents with switching topology and time-delays," IEEE Transactions on Automatic Control, vol. 49, no. 9, pp. 15201533, 2004.

[10] P. Lin and Y. Jia, "Consensus of a class of second-order multiagent systems with time-delay and jointly-connected topologies," IEEE Transactions on Automatic Control, vol. 55, no. 3, pp. 778-784, 2010.

[11] X. Wang, A. Saberi, A. A. Stoorvogel, H. v. Grip, and T. Yang, "Consensus in the network with uniform constant communication delay," Automatica, vol. 49, no. 8, pp. 2461-2467, 2013.

[12] B. Zhou and Z. Lin, "Consensus of high-order multi-agent systems with large input and communication delays," Automatica. A Journal of IFAC, the International Federation of Automatic Control, vol. 50, no. 2, pp. 452-464, 2014.
[13] L. Moreau, "Stability of continuous-time distributed consensus algorithms," in Proceedings of the 43rd IEEE Conference on Decision and Control (CDC '04), vol. 4, pp. 3998-4003, December 2004.

[14] X. Wang, A. Saberi, A. A. Stoorvoge, H. F. Grip, and T. Yang, "Consensus in the network with uniform constant communication delay," Automatica, vol. 49, no. 8, pp. 2461-2467, 2013.

[15] L. Wang and F. Xiao, "A new approach to consensus problems in discrete-time multiagent systems with time-delays," Science in China. Series F. Information Sciences, vol. 50, no. 4, pp. 625635, 2007.

[16] P. Lin, Y. Jia, and L. Li, "Distributed robust $\mathrm{H}-\mathrm{C}$ consensus control in directed networks of agents with time-delay," Systems \& Control Letters, vol. 57, no. 8, pp. 643-653, 2008.

[17] P. Lin and W. Ren, "Constrained consensus in unbalanced networks with communication delays," IEEE Transactions on Automatic Control, vol. 59, no. 3, pp. 775-781, 2014.

[18] P. Lin and Y. Jia, "Consensus of second-order discrete-time multi-agent systems with nonuniform time-delays and dynamically changing topologies," Automatica, vol. 45, no. 9, pp. 21542158, 2009.

[19] W. Ren and R. W. Beard, "Consensus seeking in multiagent systems under dynamically changing interaction topologies," Institute of Electrical and Electronics Engineers. Transactions on Automatic Control, vol. 50, no. 5, pp. 655-661, 2005.

[20] Y. Hong, L. Gao, D. Cheng, and J. Hu, "Lyapunov-based approach to multiagent systems with switching jointly connected interconnection," IEEE Transactions on Automatic Control, vol. 52, no. 5, pp. 943-948, 2007.

[21] W. Ni and D. Cheng, "Leader-following consensus of multiagent systems under fixed and switching topologies," Systems \& Control Letters, vol. 59, no. 3-4, pp. 209-217, 2010.

[22] Y.-P. Tian and C.-L. Liu, "Consensus of multi-agent systems with diverse input and communication delays," Institute of Electrical and Electronics Engineers. Transactions on Automatic Control, vol. 53, no. 9, pp. 2122-2128, 2008.

[23] W. Yang, A. L. Bertozzi, and X. Wang, "Stability of a second order consensus algorithm with time delay," in Proceedings of the 47th IEEE Conference on Decision and Control (CDC '08), pp. 2926-2931, IEEE, Cancún, Mexico, December 2008.

[24] Y. Zheng and L. Wang, "Finite-time consensus of heterogeneous multi-agent systems with and without velocity measurements," Systems and Control Letters, vol. 61, no. 8, pp. 871-878, 2012.

[25] H. Kim, H. Shim, and J. H. Seo, "Output consensus of heterogeneous uncertain linear multi-agent systems," IEEE Transactions on Automatic Control, vol. 56, no. 1, pp. 200-206, 2011.

[26] J. Wolfowitz, "Products of indecomposable, aperiodic, stochastic matrices," Proceedings of the American Mathematical Society, vol. 14, pp. 733-737, 1963. 


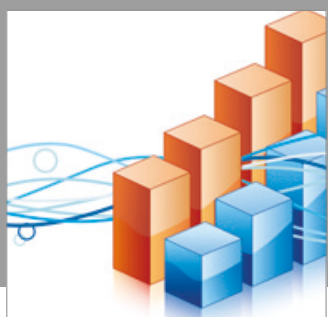

Advances in

Operations Research

vatersals

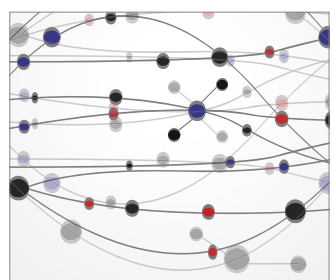

\section{The Scientific} World Journal
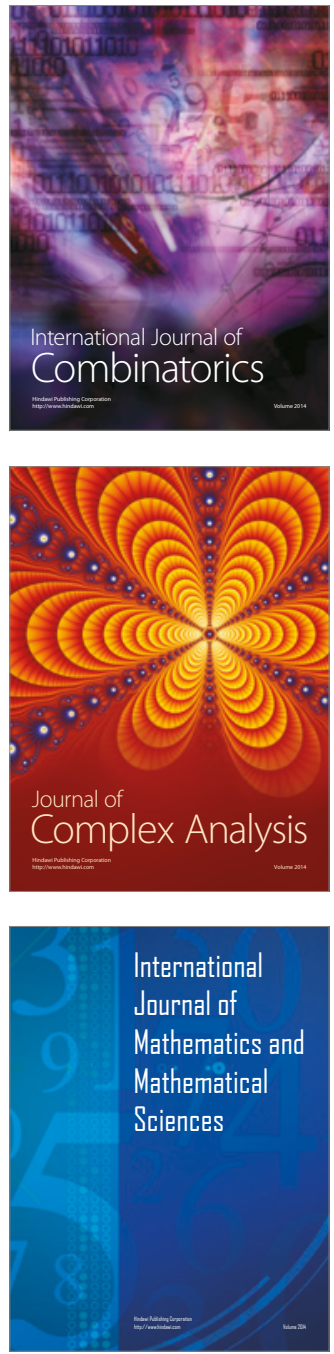
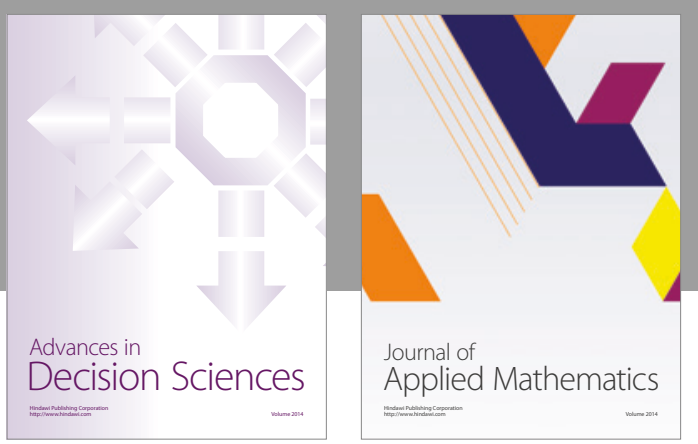

Algebra

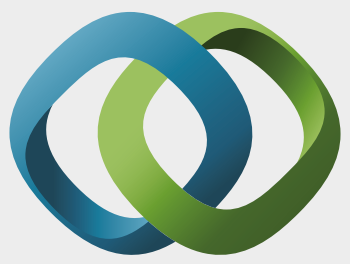

\section{Hindawi}

Submit your manuscripts at

https://www.hindawi.com
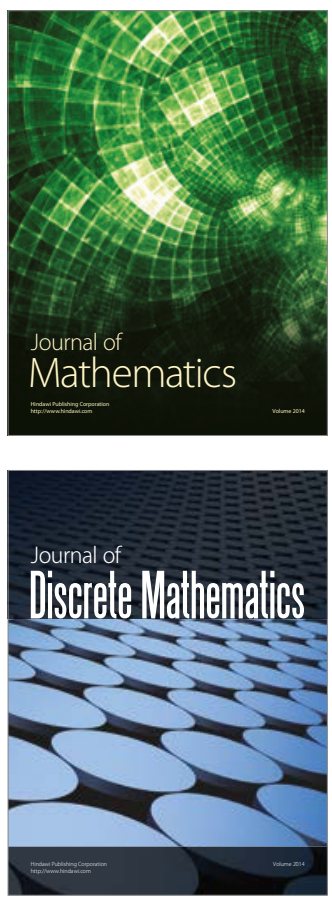

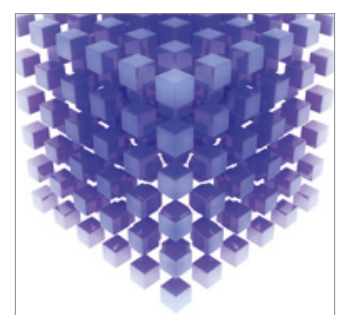

Mathematical Problems in Engineering
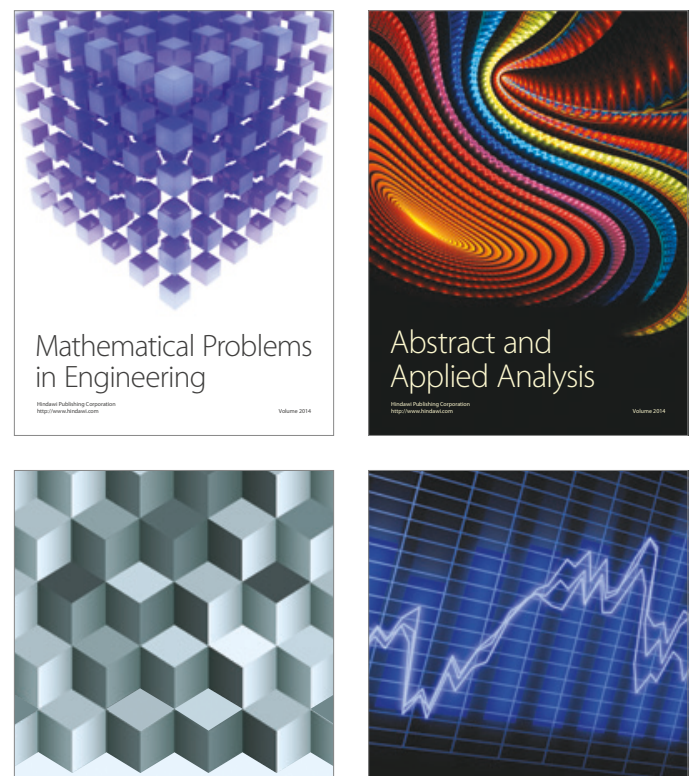

Journal of

Function Spaces

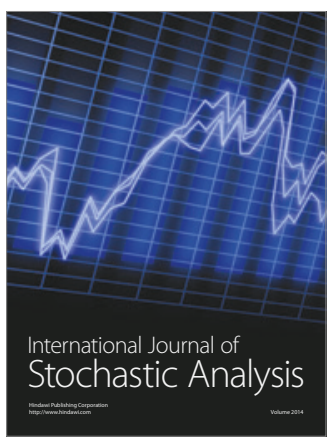

Probability and Statistics
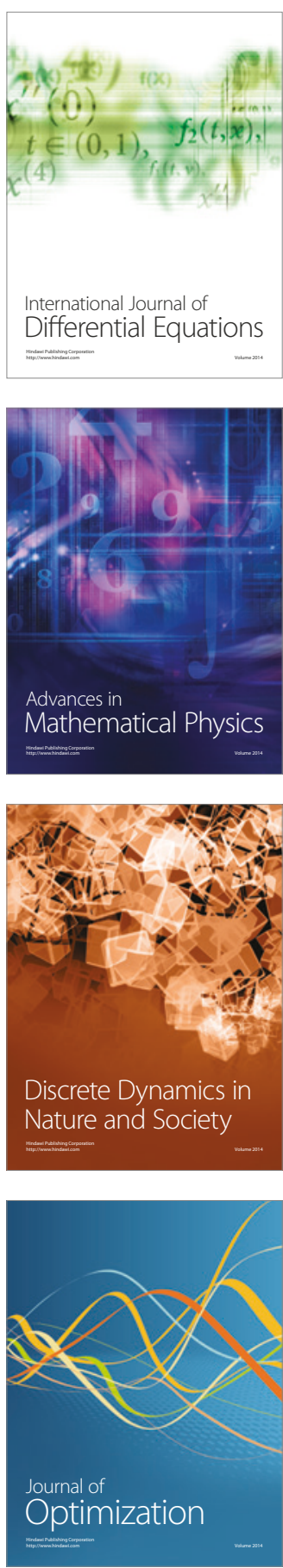